\title{
A non-destructive method for quality control of the pellet distribution within a MUPS tablet by terahertz pulsed imaging
}

\author{
Anna Novikova ${ }^{a}$ \\ Daniel Mark ${ }^{\mathbf{b}}$ \\ J. Axel Zeitlerb \\ Thomas Rades ${ }^{c}$ \\ Claudia S. Leopolda ${ }^{\mathrm{a}}$
}

a University of Hamburg, Dept. of Chemistry, Div. of Pharmaceutical Technology, Bundesstraße 45, 20146 Hamburg, Germany, anna.novikova@chemie.uni-hamburg.de, claudia.leopold@unihamburg.de

b University of Cambridge, Dept. of Chemical Engineering and Biotechnology, Philippa Fawcett Drive Cambridge, CB3 0AS, United Kingdom, dm733@cam.ac.uk, jaz22@cam.ac.uk

c University of Copenhagen, Dept. of Pharmacy, Universitetsparken 2, 2100 Copenhagen, Denmark, thomas.rades@sund.ku.dk

${ }^{*}$ Corresponding author: Prof. Dr. Claudia S. Leopold

University of Hamburg

Dept. of Chemistry

Div. of Pharmaceutical Technology

Bundesstraße 45

20146 Hamburg

Germany

Phone: +49 40 42838-3479

Fax: $\quad+494042838-6519$

e-mail address: claudia.leopold@uni-hamburg.de 


\section{ABSTRACT}

Terahertz pulsed imaging (TPI) was applied to analyse the inner structure of multiple unit pellet system (MUPS) tablets. MUPS tablets containing different amounts of theophylline pellets coated with Eudragit ${ }^{\circledR}$ NE 30 D and with microcrystalline cellulose (MCC) as cushioning agent were analysed. The tablets were imaged by TPI and the results were compared to X-ray microtomography. The terahertz pulse beam propagates through the tablets and is back-reflected at the interface between the MCC matrix and the coated pellets within the tablet causing a peak in the terahertz waveform. Cross-section images of the tablets were extracted at different depths and parallel to the tablet faces from 3D terahertz data to visualise the surface-near structure of the MUPS tablets. The images of the surface-near structure of the MUPS tablets were compared to X-ray microtomography images at the same depths. The surface-near structure could be clearly resolved by TPI at depths between 24 and $152 \mu \mathrm{m}$ below the tablet surface. An increasing amount of pellets within the MUPS tablets appears to slightly decrease the detectability of the pellets within the tablets by TPI. TPI was shown to be a fast and non-destructive method for the detection of pellets within the tablets and could resolve structures thicker than $30 \mu \mathrm{m}$. In conclusion, a proof-of-concept was provided for TPI as a method of quality control for MUPS tablets.

Key words: MUPS tablets, terahertz pulsed imaging, 3D mapping, pellet distribution analysis, spectroscopy, quality control. 


\section{INTRODUCTION}

2 Multiple unit pellet system (MUPS) tablets have great potential as solid oral dosage

3 forms, as they combine the advantages of coated single unit tablets with pellet

4 containing capsules (Abdul et al., 2010; Bodmeier, 1997). The active pharmaceutical

5 ingredient (API) in MUPS tablets is present in form of small coated pellets

6 surrounded by a tablet matrix (Collet and Moreton, 2002). MUPS tablets disintegrate

7 fast in the stomach resulting in the coated pellets to be released. These pellets pass

8 the pylorus faster and at a more predictable rate than a coated single unit tablet

9 (Bechgaard and Nielsen, 1978). A further advantage of MUPS tablets compared to

10 coated single unit tablets is their divideability without losing the pellet coating

11 functionality and thus providing high dosage flexibility (Bodmeier, 1997). However, to

12 allow dividability, a homogeneous distribution of the API pellets within the tablet

13 matrix is crucial and should be controlled and monitored during the manufacturing

14 process. As a MUPS tablet formulation often contains pellets together with powder

15 excipients, which strongly differ in terms of particle size, density and shape,

16 segregation at various steps of the manufacturing process may occur (Reich, 2005;

17 Wagner et al., 2000; Wagner et al., 1999). A fast and non-destructive method for quality control of the API pellet distribution within a MUPS tablet is thus needed.

Several spectroscopic imaging techniques allow such non-destructive analysis for 20 quality control of tablets including near-infrared (NIR) spectroscopy, Raman 21 spectroscopy, ultraviolet (UV) imaging and terahertz pulsed imaging (TPI). The NIR 22 region is defined as the range of the electromagnetic spectrum between 700 and $232500 \mathrm{~nm}\left(12820-3959 \mathrm{~cm}^{-1}\right)$ and NIR spectroscopy is a well investigated method for 24 various pharmaceutical applications (Reich, 2005). Moreover, NIR spectroscopy has 25 been demonstrated to be a powerful imaging tool for monitoring the API content 
uniformity and API distribution in single unit tablets (Amigo and Ravn, 2009; Cruz and

27 Blanco, 2011; Franch-Lage et al., 2011; Lee et al., 2006; Palou et al., 2012). Zhao et al. applied NIR mapping to visualize the distribution of metoprolol succinate pellets on the surface of commercially available MUPS tablets (Zhao et al., 2010). Raman spectroscopy is another frequently applied analytical method for the characterisation of pharmaceutical formulations. It is based on the inelastic scattering of light, often in the same range of energy as infrared spectroscopy (Gordon and McGoverin, 2011). The application of Raman imaging to analyse the tablet ingredient distribution within single unit tablets is extensively described in the literature (Boiret et al., 2014; Firkala et al., 2013, Sasić, 2007a, 2007b, Slipchenko et al., 2010; Vajna et al., 2011). The application of Raman imaging to analyse the structure of MUPS tablets has not yet been described in the literature, but is also theoretically possible. UV imaging has also been shown to be applicable for the visualisation of the distribution of theophylline pellets on the tablet surface (Novikova et al., 2016a). However, UV radiation does not provide a high penetration depth into a tablet. Therefore, only pellets directly at the tablet surface are considered in the analysis. With the described imaging techniques, information on structures below the tablet surface can only be obtained by destructive and time consuming handling of the tablet (Shen and Taday, 2008).

In the present study, terahertz pulsed imaging (TPI) is evaluated as a quality control tool for MUPS tablets. Terahertz radiation is defined as the range of the

47 electromagnetic spectrum between microwaves and infrared radiation $\left(2-133 \mathrm{~cm}^{-1}\right.$, 0.1-4 THz) (Zeitler et al., 2007a). Terahertz radiation propagates through most pharmaceutical materials, allowing a high penetration depth and thus analysis of the 
51 et al., 2007a). Therefore, terahertz radiation has gained interest in pharmaceutical

52 research for analysis of solid dosage forms with complex inner structures (Shen and

53 Taday, 2008). The interfaces separating physical structures with different refractive

54 indices, such as interfaces between pellets and the matrix in a MUPS tablet, are

55 visible as peaks in the measured terahertz waveform. Therefore, these features in

56 the terahertz waveform allow the detection of interfaces below the sample surface.

57 The main applications of TPI in the pharmaceutical field are the analysis of the 58 coating thickness (Haaser et al., 2013) and its uniformity (Zeitler and Shen, 2013) as

59 well as in-line monitoring of coating processes (May et al., 2011). The application of

60 TPI for chemical imaging as well as for API quantification has also been discussed in

61 the literature (Cogdill et al., 2006; Shen et al., 2005a). Compared to NIR and Raman

62 imaging, TPI allows the extraction of chemical and physical information on the

63 different depths within a sample simultaneously (3D imaging) (Zeitler and Shen,

64 2013). Therefore, it was possible to perform chemical imaging of lactose and tartaric

65 acid with TPI in depth within a model tablet (Shen et al., 2005b). However, this model

66 tablet consisted of polyethylene, which is completely transparent to terahertz

67 radiation and therefore scattering of the terahertz radiation within the tablet was

68 minimised (Shen et al., 2005b; Zeitler and Shen, 2013). Nevertheless, this method

69 holds great potential for chemical imaging within a tablet.

70 The suitability of TPI for the analysis of the inner structure of MUPS tablets was

71 investigated in this study. For this purpose, MUPS tablets containing varying

72 amounts of theophylline pellets coated with Eudragit ${ }^{\circledR} \mathrm{NE} 30 \mathrm{D}$ were compressed

73 with microcrystalline cellulose (MCC) as a cushioning agent and thereafter analysed

74 by TPI. The effect of the depth resolution limit and the influence of the coated pellet

75 amount in the MUPS tablets on the detectability of the pellets below the tablet 
76 surface was investigated. The TPI results were compared to X-ray microtomography 77 (microCT) measurements as reference method. 


\section{MATERIALS AND METHODS}

\subsection{Materials}

80

Theophylline matrix core pellets obtained by extrusion and spheronisation containing

$8196.5 \%$ theophylline, were supplied by Temmler (Killorglin, Ireland).

82 Eudragit $^{\circledR}$ NE 30 D was donated by Evonik (Darmstadt, Germany). MCC (Ceolus ${ }^{\circledR}$

83 KG-802) was a gift by Asahi Kasei Chemicals (Tokyo, Japan). Hydroxypropyl 84 methylcellulose (HPMC; Pharmacoat ${ }^{\circledR}$ 603) was purchased from Harke Pharma 85 (Mülheim an der Ruhr, Germany), polysorbate 80 was obtained from Caelo (Hilden, 86 Germany), and talc from Fagron (Barsbüttel, Germany).

\subsection{Methods}

\subsubsection{Sample preparation}

90 To obtain coated theophylline pellets for manufacturing of MUPS tablets, a 91 Eudragit $^{\circledR}$ NE coating dispersion was prepared by homogenising HPMC as a gelling 92 agent in purified water at $40{ }^{\circ} \mathrm{C}$ using an Ultra Turrax ${ }^{\circledR}$ (IKA, Staufen, Germany). After 93 a solution was formed, polysorbate 80 as a plasticiser and talc as anti-tacking agent 94 were added and dispersed for at least 10 min and subsequently slowly poured into 95 the Eudragit ${ }^{\circledR}$ NE 30 D dispersion under continuous stirring with a propeller stirrer 96 (Eurostar 100 digital, IKA, Staufen, Germany) for at least $5 \mathrm{~min}$. The resulting coating 97 dispersion contained $22.7 \%$ Eudragit $^{\circledR}$ NE 30 D, $6.8 \%$ talc, $0.7 \%$ polysorbate 80 , and $980.7 \%$ HPMC (w/w). After preparation of the coating dispersion, $400 \mathrm{~g}$ of theophylline 99 pellets (mesh $900-1000 \mu \mathrm{m}$ ) were coated in a bottom spray fluidised bed apparatus

100 (Solidlab 1, Bosch Packaging Technology, Schopfheim, Germany). The coating 101 parameters were adjusted as follows: inlet air temperature $16{ }^{\circ} \mathrm{C}$; inlet air flow rate $10235 \mathrm{~m}^{3} \mathrm{~h}^{-1}$; atomising air pressure 1.5 bar; microclimate 0.4 bar; spraying rate 
$1031.5-3.0 \mathrm{~g} \mathrm{~min}^{-1}$. The nozzle diameter was $0.8 \mathrm{~mm}$. The obtained coated pellets were 104 subsequently dried in an oven at $40{ }^{\circ} \mathrm{C}$ for $48 \mathrm{~h}$. The total coating applied to the pellets amounted to a $5 \%$ weight gain in polymer mass.

106 The coated theophylline pellets were mixed with MCC powder to obtain five batches 107 of tablets with varying amounts of pellets $(30 \%, 40 \%, 50 \%, 60 \%$, and $70 \%(w / w))$. $108250 \mathrm{mg}$ of each formulation were manually filled into the die and compacted at $109255 \mathrm{MPa}$ using the single punch mode of an instrumented rotary press (Fette 102i, 110 Fette Compacting, Schwarzenbek, Germany), equipped with $10 \mathrm{~mm}$ flat-faced 111 punches, resulting in tablets between 2.2 and $2.4 \mathrm{~mm}$ thickness. The tablet thickness 112 and tensile strength were determined with a tablet hardness tester (Erweka TBH425, 113 Heusenstamm, Germany). The tensile strength of the produced tablets was above 3 $114 \mathrm{MPa}$ to ensure tablets of significant hardness comparable to industrially produced 115 tablets.

\subsubsection{Terahertz pulsed imaging}

117 Five MUPS tablets, one from each batch, were imaged with a commercial TPI system 118 (TPI ${ }^{\mathrm{TM}}$ imaga 2000, TeraView, Cambridge, UK) which represents an automated 119 tablet scanner. As shown in Fig. 1 the TPI system scans across the $x$ - and $y$ 120 direction of the top and bottom face of the sample tablets and thereby records single 121 depth profiles at $200 \mu \mathrm{m}$ lateral resolution. The scanning procedure is based on a 3D 122 model of the surface, which is generated prior to the terahertz measurements. This $1233 \mathrm{D}$ model is further required for analysis of the terahertz data to locate each 124 waveform and thus to enable the 3D reconstruction of the sample. Each terahertz waveform within a sample tablet represents a depth profile equivalent to $3.5 \mathrm{~mm}$ pulse propagation in air (refractive index, $n=1$ ). The actual penetration depth is

127 dependent on the actual refractive index of the tablet matrix as well as the absorption 
128 of the terahertz pulse by the matrix. Each 3D measurement thus covered a volume of $1299.7 \times 9.7 \times 3.5 \mathrm{~mm}^{3}(49 \times 49 \times 512$ pixels $)$. The scheme of the resulting terahertz 130 waveform in Fig. 1 shows the peaks originating from the reflection at the front face of 131 the tablet as well as at the interfaces of the surface-near pellets. As the average 132 thickness of the pellets' coating layers is below the depth resolution of the used TPI $133(\approx 35 \mu \mathrm{m})$, only two peaks per pellet are observed (e.g. labelled with 2,3 or 4,5 in 134 Fig. 1) even though the coating and the pellet core have different refractive indices.

135 The data acquisition time for one tablet face was $25 \mathrm{~min}$. Analysis of the images was 136 performed with Matlab (ver. 8.1, Mathworks, Natick, USA). Wavelet denoising was 137 applied on each terahertz waveform using 4-layer Daubechies wavelets and 138 performing the wavelet decomposition at level 8 . This procedure helped to highlight 139 inner structures and supressed noise in the terahertz waveforms.

\subsubsection{X-ray microtomography}

141 The same MUPS tablets that were analysed by TPI were scanned with X-ray 142 microtomography (Skyscan 1172, Bruker microCT, Kontich, Belgium) applying a 143 source voltage of $59 \mathrm{kV}$. The tablets were rotated during the measurement, and 803 144 transmission images were recorded in steps of $0.25^{\circ}$. The exposure time for each 145 transmission image was $780 \mathrm{~ms}$. The scan duration for one tablet varied between 43 146 and 53 min. Reconstruction of the microCT images was performed with NRecon 147 software (ver. 1.6.8, Bruker microCT, Kontich, Belgium) and further analysis of the 148 images was conducted with the Dataviewer software (ver. 1.5.2, Bruker microCT, 149 Kontich, Belgium). The isotropic voxel size of the reconstructed images varied 150 between $3.04 \mu \mathrm{m}$ and $3.98 \mu \mathrm{m}$. 


\section{RESULTS AND DISCUSSION}

152 In the present study, the suitability of TPI to analyse the inner structure of MUPS 153 tablets was investigated. The signals caused by propagation of the terahertz 154 radiation through the MUPS tablets were correlated with the physical structure of the 155 tablets. The pulse of terahertz radiation propagates through the tablet and is partly 156 reflected by interfaces of structures with different refractive indices (Zeitler and Shen, 157 2013). The time delay $\Delta t$ between the reflections of two different interfaces can be measured and used to calculate the actual depth $d=\Delta t c / 2 n$, where $n$ is the refractive index of the medium and $c$ is the speed of light (Shibuya and Kawase, 2013). A sample terahertz waveform resulting from the reflection of the terahertz 161 pulse at the interface of the structures in a MUPS tablet containing $30 \%(\mathrm{w} / \mathrm{w})$ of 162 coated theophylline pellets is presented in Fig. 2a. The terahertz electric field is plotted versus the time delay relative to the reflection from the surface of the MUPS 164 tablet (corresponds to $0 \mu \mathrm{m}$ time delay). The units are propagation distance of the 165 equivalent length of travel in air $(n=1)$. The first reflection peak (1) is caused by the 166 terahertz pulse being reflected at the tablet surface in the matrix area. Thereafter, the terahertz pulse propagates into the tablet matrix and is reflected at the interface between the tablet matrix (MCC) and a coated pellet resulting in a second peak (2).

169 The contact region between the tablet matrix and the pellet includes both an interface

170 between the tablet matrix and the coating and an interface between the coating and 171 the pellet core. However, the coating (coating level 5\%) is thinner than the TPI depth 172 resolution limit of about $35 \mu \mathrm{m}$ (Haaser et al., 2013). Thus, the reflections at these 173 interfaces result only in a single peak representing a "combined" interface caused by 174 the short time delay between these signals. After the reflection at this "combined" 175 interface, the terahertz pulse further propagates into the theophylline pellet until it 
once again is reflected at the "combined" interface between the pellet and the tablet matrix resulting in a third peak (3).

Another example of the waveform caused by the terahertz pulse that propagates into the MUPS tablet is outlined in Fig. $2 \mathrm{~b}$. The first peak (4) is again caused by terahertz pulse reflection at the surface of the tablet. In this case, however, the signal for the reflection of the terahertz pulse at the interface between the tablet matrix and pellet core is not detectable because this particular pellet is too close to the tablet surface and its signal is overlapped by the first peak. After that reflection, the terahertz pulse further propagates into the theophylline pellet. Subsequently, the second peak (5) is the result of the reflection at the interface between the pellet core and the tablet matrix. This described waveform is presented for a pellet, which is located close to the surface and has lost its spherical shape during compression.

As the measured terahertz waveform is the result of the convolution of the incident terahertz pulse and the impulse response function of the sample, features of the incident terahertz pulse (e.g. negative peaks) are also visible in the measured waveform. In the majority of cases, this is corrected by a deconvolution using a reference waveform (e.g. the reflection measurement of a mirror). Such deconvolution amplifies high-frequency noise, which can be reduced by applying a filter. In the present study a deconvolution was applied and different filters (double Gaussian and Wiener filter) were tested, which improved the overall signal to noise ratio. However, the filters introduced artificial features causing a misdetection of pellets and it suppressed characteristic peaks originating from the pellet interfaces. Therefore, the raw terahertz waveforms were used for comparison with the microCT data. 
200 For better visualisation of the 3D structure of the MUPS tablet with a theophylline 201 pellet amount of 30\% (w/w), microCT and TPI images at six different depths $(24 \mu \mathrm{m}$, $20249 \mu \mathrm{m}, 73 \mu \mathrm{m}, 97 \mu \mathrm{m}, 128 \mu \mathrm{m}$ and $152 \mu \mathrm{m})$ below the tablet surface are presented in 203 Fig. 3. In the microCT greyscales images, the pellets are visualised by darker grey 204 colour, as a result of lower density, compared to the MCC matrix. The colours in the

205 TPI images represent the strength of the terahertz electric field. In this context, the 206 yellow colour indicates a high electric field and blue colour - a low one. In this proof of 207 concept study, the coated theophylline pellets embedded in the MCC matrix can be 208 identified in the TPI images up to a depth of $152 \mu \mathrm{m}$. Although the terahertz radiation 209 penetrates through the entire tablet, the contrast of the pellets' interfaces is very low 210 at depths $>152 \mu \mathrm{m}$ because of the used optics (i.e. the focal point is at the surface of 211 the tablet) and scattering losses. A yellow colour of the pixels corresponds to a high 212 terahertz electric field indicating that the terahertz pulse reaches the surface of the 213 pellet within the tablet and is reflected at this interface as described above. The first 214 cross-section image was analysed at the depth of $24 \mu \mathrm{m}$. This depth resolution limit 215 was determined by comparison of the first TPI cross-section image with the microCT 216 data. As expected, the results show that the signals caused by the back-reflection of 217 the terahertz pulse from the internal interface still overlap with the signal of the back218 reflected terahertz pulse from the surface of the tablet.

219 The pellets that can be detected based on the TPI data in the first image of Fig. 3 are 220 marked with a red "1". The TPI results for the pellet distribution at the depth of $24 \mu \mathrm{m}$ 221 in the tablet were confirmed by the microCT investigation. The depth position of the 222 pellets below the surface determined by TPI may slightly differ (several $\mu \mathrm{m}$ ) from that 223 determined by microCT. This deviation is primarily attributed to the different refractive 224 indices of MCC, the Eudragit ${ }^{\circledR}$ coating of the pellets and the theophylline pellets 
225 causing variations in the propagation velocity of the terahertz pulse while it 226 propagates through the tablet. In addition to that, it was difficult to accurately 227 superimpose both datasets in 3D.

228 In the second image of Fig. 3 (depth of $49 \mu \mathrm{m}$ ) a change of the yellow colour to green 229 or blue for various pellets that are marked with "1" can be observed. This change 230 results from the decrease of the terahertz electric field after the peak maximum. 231 However, the differentiation between various pellets that are marked with "1" and the 232 tablet matrix is impossible at this depth because there is no interface present. 233 Therefore, to analyse the pellet distribution deep below the tablet surface of a MUPS 234 tablet, the TPI images at several depths should be analysed instead of TPI image at 235 one preselected depth. Pellets which were first detectable at the depth of $49 \mu \mathrm{m}$ are 236 marked with "2" (Fig. 3, image 2).

237 The pellets contain $96.5 \%$ of theophylline and are coated with a flexible polymer 238 (please refer to section 2.1), resulting in soft pellets, which have lost their spherical 239 structure during compression. Therefore, the shape of the pellets may vary 240 depending on their location below the surface of the MUPS tablet. Thus, several 241 interface spots between the pellet and the matrix exist for one pellet at varying 242 depths. The terahertz pulse is reflected at these interfaces resulting in a high 243 terahertz electric field (yellow colour) for one pellet at varying depths. This can be 244 observed for the pellets marked with "1" in the lower left corner of the TPI images. 245 These pellets are characterised by a high terahertz electric field in the first image and 246 then again especially in the fourth image. As described above, the detectable 247 interface spots of these pellets increase at the depth of $97 \mu \mathrm{m}$ leading to new 248 interfaces resulting in back-reflection of the terahertz pulses and therefore in high 249 terahertz electric field values (yellow colour). 
250 In a previously performed study on the determination of the coating thickness of 251 coated tablets with TPI, it was possible to analyse the inner tablet structure up to a 252 depth of $300 \mu \mathrm{m}$ (Novikova et al., 2016b). In contrast, in the present study 253 investigating MUPS tablets it was only possible to detect pellet structures up to $254152 \mu \mathrm{m}$ below the tablet surface (Fig. 3), because of high scattering losses and the 255 high density of the MUPS tablet components compared to the coating of coated 256 tablets. Therefore, the location of small objects such as pellets at greater depth is 257 difficult, as the divergence of the terahertz beam as well as scattering and absorption 258 losses will further decrease the signal contrast. Nevertheless, the analysis of depths $259>152 \mu \mathrm{m}$ below the tablet surface appears still feasible, because Zeitler et al. 260 demonstrated that internal interfaces up to $2 \mathrm{~mm}$ below the tablet surface of coated 261 tablets can be detected (Zeitler et al., 2007b).

262 To determine the influence of the pellet amount in the MUPS tablets on the 263 detectability of the pellets embedded in the MCC matrix, tablets with different 264 amounts of pellets (expressed as the weight percentage of pellets in the MUPS 265 tablet) were investigated. As shown in Fig. 3 for a MUPS tablet with a pellet amount 266 of $30 \%(w / w)$, all pellets which were detectable in the microCT images were also 267 detected in the TPI images. In Fig. 4 microCT and TPI images of four MUPS tablets 268 with a pellet amount between 40 and $70 \%(\mathrm{w} / \mathrm{w})$ are displayed. The images are 269 presented only at one selected depth per tablet. However, to detect as many pellets 270 as possible, images at depths below the selected depths were also analysed (data 271 not shown). Pellets detected in the TPI images at depths below the selected depths 272 are marked with "1". Furthermore, pellets detected in the TPI images of the selected 273 depths are marked with "2". In the image of the MUPS tablet with a pellet amount of $27440 \%(w / w)$ all pellets which were present in the microCT image, were also detected 
275 in the TPI image. In the microCT image of the MUPS tablet with a pellet amount of $27650 \%$, two pellets (marked with "0") were found, which were undetectable in the TPI 277 images (Fig. 4b). Interestingly, these pellets were visible with microCT on the tablet 278 surface. Thus, the peak caused by the back-reflection of the terahertz pulse at the 279 interface between the pellet and the matrix may have overlapped with that at the 280 interface between air and tablet surface (matrix), or this surface peak is already 281 caused by the back-reflection at the interface between air and pellet surface. The two 282 undetected pellets are located close to the edge of the tablet. In the TPI image of the 283 MUPS tablet with a pellet amount of $60 \%(\mathrm{w} / \mathrm{w})$ again two pellets (marked with "0"), 284 which were also located close to the edge of the tablet, were not found in the TPI 285 images. Apparently, edge effects are occurring in the terahertz waveforms acquired 286 close to the tablet edge because of a diffraction-limited focal spot of about $200 \mu \mathrm{m}$. 287 As shown in Fig. 4d, for the MUPS tablet with a pellet amount of $70 \%(w / w)$ the 288 number of undetected pellets increases to six (marked with "0"), compared to the 289 MUPS tablets with lower pellet amounts. Three of the undetected pellets were again 290 located close to the tablet edge confirming the assumption that the applied optics 291 decreases the pellet detectability at the edge of the tablet. The other three of the 292 undetected pellets were not located close to the tablet edge, but are already visible 293 on the tablet surface. This confirms the hypothesis that the back-reflection peak of 294 the terahertz pulse beam at the interface between matrix and pellets which are visible 295 on the tablet surface may overlap with the back-reflection peak of the terahertz pulse 296 at the tablet surface. However, in general, the detectability of pellets based on the 297 TPI images appears to decrease slightly with increasing pellet amount in the MUPS 298 tablet. Nevertheless, it should be mentioned that these pellets may still be detected 299 based on terahertz electric field values at other depths below the tablet surface. 
300 In Table 1 the results regarding the number of pellets detected in the TPI and 301 microCT images of MUPS tablets up to the depths presented in Fig. 4 are 302 summarized. For better comparison of the results for the different pellet amounts 303 within the MUPS tablets the percentage of the number of pellets detected in the TPI 304 images with regard to the actual number of visible pellets in the microCT images was 305 calculated. For tablets with a low pellet amount (30 and $40 \%(w / w))$ all pellets that 306 were visible in the microCT images, could also be detected in the TPI images. In the 307 TPI images of tablets with pellet amounts of at least $50 \%(\mathrm{w} / \mathrm{w})$ more than $87 \%$ of 308 pellets that were visible with microCT, could be detected.

309 Table 1: Comparison of the number of pellets detected in MUPS tablets with 310 varying pellet amounts by microCT and TPI.

\begin{tabular}{lccccc}
\hline $\begin{array}{l}\text { Pellet amount in MUPS } \\
\text { tablet: }\end{array}$ & $30 \%(\mathrm{w} / \mathrm{w})^{1}$ & $40 \%(\mathrm{w} / \mathrm{w})^{2}$ & $50 \%(\mathrm{w} / \mathrm{w})^{2}$ & $60 \%(\mathrm{w} / \mathrm{w})^{2}$ & $70 \%(\mathrm{w} / \mathrm{w})^{2}$ \\
\hline $\begin{array}{l}\text { Number of pellets detected } \\
\text { in microCT images }\end{array}$ & 18 & 16 & 19 & 25 & 45 \\
\hline $\begin{array}{l}\text { Number of pellets detected } \\
\text { in TPI images }\end{array}$ & 18 & 16 & 17 & 23 & 39 \\
\hline $\begin{array}{l}\text { Percentage of pellets } \\
\text { detected by TPl compared } \\
\text { to microCT }\end{array}$ & $100 \%$ & $100 \%$ & $89 \%$ & $92 \%$ & $87 \%$ \\
\hline
\end{tabular}

$311{ }^{1}$ Number of pellets detected up to the depth of $49 \mu \mathrm{m}$ below the tablet surface.

$312{ }^{2}$ Number of pellets detected up to the selected depth below the tablet surface.

313 In general, it can be stated that it was possible to detect most of the pellets in the TPI

314 images up to the selected depth regardless of the pellet amount in the MUPS tablets 315 and to confirm these results with the microCT images. TPI has been shown to be a 316 faster and safe method than microCT, with a pixel size in depth of $4.9 \mu \mathrm{m}$ and the 317 possibility to precisely resolve structures thicker than about $30-40 \mu \mathrm{m}$ for the 318 analysis of the surface-near structure of MUPS tablets. 
319 The dividability is an advantage of MUPS tablets. Therefore, rapid and non320 destructive methods for the evaluation of the pellet distribution within the MUPS 321 tablet are needed. The advantage of TPI compared to surface imaging methods is 322 the possibility to obtain additional information on the pellet distribution below the 323 tablet surface. As already mentioned, with the optics of the TPI device it was possible 324 to analyse the pellet distribution up to $152 \mu \mathrm{m}$ below the tablet surface. Novikova et 325 al. investigated the suitability of UV imaging for analysis of the pellet distribution on 326 the MUPS tablet surface (Novikova et al., 2016a). In this study it could be shown that 327 the pellet amount in a MUPS tablet can be estimated based on the amount of pellets 328 determined on the tablet surface. In addition, the pellet amount in a tablet half after 329 tablet division could be estimated based on the pellet amount determined on the 330 surface of this tablet half. Based on the data of the present study, the determination 331 of the pellet amount within a MUPS tablet by TPI analysis of the tablet appears to be 332 more suitable than a surface method (e.g. UV imaging). Especially for tablets with a 333 low pellet amount and, thus also a low number of detectable pellets at the tablet 334 surface, estimation of the pellet amount in the tablets based on TPI images may 335 improve the quality of the determination. Additionally, Novikova et al. showed that for 336 thicker tablets the estimation of the pellet amount within a tablet based on the tablet 337 surface analysis by a UV imager was less precise than for the thinner tablets 338 (Novikova et al., 2016a).

339 NIR mapping has also been shown to be applicable for visualization of the pellet 340 distribution on the surface of MUPS tablets (Zhao et al., 2010). Nevertheless, NIR, 341 such as NIR mapping in reflection, is a surface-biased method and may not be 342 applicable for tablets with a low amount of pellets on the tablet surface or for thicker 343 MUPS tablets. As described in the introduction, Raman imaging has not yet been 
344 applied for analysis of the pellet distribution within MUPS tablets. Moreover, Raman

345 is also a surface biased technique. Therefore, depth information may only be 346 obtained by microtoming the samples and successively imaging every plane. 


\section{CONCLUSION}

348 In this study, the suitability of TPI for the detection of coated theophylline pellets 349 within a MUPS tablet was investigated. It was found, that a pellet in a tablet causes 350 two peaks: one as soon as the terahertz pulse is back-reflected at the interface 351 between the tablet matrix and the pellet surface and a second when the terahertz 352 pulse leaves the pellet and is reflected at the interface between the pellet and the 353 matrix. Thus, TPI allows the visualisation of the interfaces between the pellets and 354 the tablet matrix. The first evaluable cross-section TPI images were achieved at $35524 \mu \mathrm{m}$ below the tablet surface. With the applied optics it was possible to detect the 356 pellets in the TPI images up to at least $152 \mu \mathrm{m}$ below the tablet surface. Increasing 357 the amount of pellets within the MUPS tablets appeared to slightly decrease the 358 pellet detectability. The undetected pellets were located close to the tablet edge or at 359 the tablet surface. In conclusion, TPI was shown to be a promising technique for fast 360 and non-destructive analysis with a high depth resolution within the MUPS tablets. 361 Therefore, the present study serves as a proof-of-concept for quality control of MUPS 362 tablets by means of TPI. 


\section{ACKNOWLEDGMENTS}

364 The authors would like to thank Evonik for providing Eudragit ${ }^{\circledR}$ NE 30D as well as

365 Asahi Kasei Chemicals for supplying MCC. This research did not receive any specific

366 grant from funding agencies in the public, commercial, or not-for-profit sectors. 


\section{REFERENCES}

Abdul, S., Chandewar, A.V., Jaiswal, S.B., 2010. A flexible technology for modifiedrelease drugs: Multiple-unit pellet system (MUPS). J. Control. Release 147 (1), 216.

Amigo, J.M., Ravn, C., 2009. Direct quantification and distribution assessment of major and minor components in pharmaceutical tablets by NIR-chemical imaging. Eur. J. Pharm. Sci. 37 (2), 76-82.

Bechgaard, H., Nielsen, G.H., 1978. Controlled-release multiple-units and single-unit doses a literature review. Drug Dev. Ind. Pharm. 4 (1), 53-67.

Bodmeier, R., 1997. Tableting of coated pellets. Eur. J. Pharm. Biopharm. 43 (1), $1-$ 8.

Boiret, M., de Juan, A., Gorretta, N., Ginot, Y.-M., Roger, J.-M., 2015. Distribution of a low dose compound within pharmaceutical tablet by using multivariate curve resolution on Raman hyperspectral images. J. Pharm. Biomed. Anal. 103, 35-43.

Cogdill, R.P., Short, S.M., Forcht, R., Shi, Z., Shen, Y., Taday, P.F., Anderson, C.A., Drennen, J.K., 2006. An efficient method-development strategy for quantitative chemical imaging using terahertz pulse spectroscopy. J. Pharm. Innov. 1 (1), 6375.

Collet, J., Moreton, C., 2002. Modified release peroral dosage forms, in: Aulton, M.E. (Ed.), Pharmaceutics. The science of dosage form design, second ed. Churchill Livingstone, Edinburgh, pp. 289-305.

Cruz, J., Blanco, M., 2011. Content uniformity studies in tablets by NIR-Cl. J. Pharm. Biomed. Anal. 56 (2), 408-412.

Firkala, T., Farkas, A., Vajna, B., Farkas, I., Marosi, G., 2013. Investigation of drug distribution in tablets using surface enhanced Raman chemical imaging. J. Pharm. Biomed. Anal. 76, 145-151.

Fitzgerald, A.J., Cole, B.E., Taday, P.F., 2005. Nondestructive analysis of tablet coating thicknesses using terahertz pulsed imaging. J. Pharm. Sci. 94 (1), 177183.

Franch-Lage, F., Amigo, J.M., Skibsted, E., Maspoch, S., Coello, J., 2011. Fast assessment of the surface distribution of API and excipients in tablets using NIRhyperspectral imaging. Int. J. Pharm. 411 (1-2), 27-35.

Gordon, K.C., McGoverin, C.M., 2011. Raman mapping of pharmaceuticals. Int. J. Pharm. 417 (1-2), 151-162.

Haaser, M., Gordon, K.C., Strachan, C.J., Rades, T., 2013. Terahertz pulsed imaging as an advanced characterisation tool for film coatings-A review. Int. J. Pharm. 457 (2), 510-520.

Lee, E., Huang, W.X., Chen, P., Lewis, E.N., Vivilecchia, R.V., 2006. High-throughput analysis of pharmaceutical tablet content uniformity by near-infrared chemical imaging. Spectrosc. 21 (11), 24-32.

May, R.K., Evans, M.J., Zhong, S., Warr, I., Gladden, L.F., Shen, Y., Zeitler, J.A., 2011. Terahertz in-line sensor for direct coating thickness measurement of individual tablets during film coating in real-time. J. Pharm. Sci. 100 (4), 15351544.

Novikova, A., Carstensen, J.M., Rades, T., Leopold, C.S., 2016a. Multispectral UV imaging for surface analysis of MUPS tablets with special focus on the pellet distribution. Int. J. Pharm 515 (1), 374-383. 
Novikova, A., Carstensen, J.M., Zeitler, J.A., Rades, T., Leopold, C.S., 2016b. Multispectral UV imaging for determination of the tablet coating thickness. J. Pharm. Sci. https://doi.org/10.1016/j.xphs.2017.02.016

Palou, A., Cruz, J., Blanco, M., Tomas, J., de los Rios, J., Alcala, M., 2012. Determination of drug, excipients and coating distribution in pharmaceutical tablets using NIR-Cl. J. Pharm. Anal. 2 (2), 90-97.

Reich, G., 2005. Near-infrared spectroscopy and imaging: Basic principles and pharmaceutical applications. Adv. Drug Deliv. Rev. 57 (8), 1109-1143.

Sasić, S., 2007a. An in-depth analysis of Raman and near-infrared chemical images of common pharmaceutical tablets. Appl. spectrosc. 61 (3), 239-250.

Sasić, S., 2007b. Raman mapping of low-content API pharmaceutical formulations. I. Mapping of alprazolam in alprazolam/xanax tablets. Pharm. Res. 24 (1), 58-65.

Shen, Y.C., Taday, P.F., Newnham, D.A., Pepper, M., 2005a. Chemical mapping using reflection terahertz pulsed imaging. Semicond. Sci. Technol. 20 (7), S254S257.

Shen, Y.-C., 2011. Terahertz pulsed spectroscopy and imaging for pharmaceutical applications: A review. Int. J. Pharm 417 (1-2), 48-60.

Shen, Y.-C., Hwu, R.J., Linden, K.J., Taday, P.F., Newnham, D.A., Kemp, M.C., Pepper, M., 2005b. 3D chemical mapping using terahertz pulsed imaging, Proc. SPIE 5727, Terahertz and Gigahertz Electronics and Photonics IV, 24. doi:10.1117/12.591472

Shen, Y.-C., Taday, P.F., 2008. Development and application of terahertz pulsed imaging for nondestructive inspection of pharmaceutical tablet. IEEE J. Sel. Top. Quantum Electron. 14 (2), 407-415.

Shibuya, T., Kawase, K., 2013. THz Tomography, in: Peiponen, K.-E., Zeitler, A., Kuwata-Gonokami, M. (Eds.), Terahertz spectroscopy and imaging. Springer, Heidelberg, pp. 433-449.

Slipchenko, M.N., Chen, H., Ely, D.R., Jung, Y., Carvajal, M.T., Cheng, J.-X., 2010. Vibrational imaging of tablets by epi-detected stimulated Raman scattering microscopy. Analyst 135 (10), 2613-2619.

Vajna, B., Patyi, G., Nagy, Z., Bódis, A., Farkas, A., Marosi, G., 2011. Comparison of chemometric methods in the analysis of pharmaceuticals with hyperspectral Raman imaging. J. Raman Spectrosc. 42 (11), 1977-1986.

Wagner, K.G., Krumme, M., Beckert, T.E., Schmidt, P.C., 2000. Development of disintegrating multiple-unit tablets on a high-speed rotary tablet press. Eur. J. Pharm. Biopharm. 50 (2), 285-292.

Wagner, K.G., Krumme, M., Schmidt, P.C., 1999. Investigation of the pelletdistribution in single tablets via image analysis. Eur. J. Pharm. Biopharm. 47 (1), 79-85.

Zeitler, J.A., Taday, P.F., Newnham, D.A., Pepper, M., Gordon, K.C., Rades, T., 2007a. Terahertz pulsed spectroscopy and imaging in the pharmaceutical setting A review. J. Pharm. Pharmacol. 59 (2), 209-223.

Zeitler, J.A., Shen, Y., Baker, C., Taday, P.F., Pepper, M., Rades, T., 2007b. Analysis of coating structures and interfaces in solid oral dosage forms by three dimensional terahertz pulsed imaging. J. Pharm. Sci. 96 (2), 330-340.

Zeitler, J.A., Shen, Y.-C., 2013. Industrial applications of terahertz imaging, in: Peiponen, K.-E., Zeitler, A., Kuwata-Gonokami, M. (Eds.), Terahertz spectroscopy and imaging. Springer, Heidelberg, pp. 451-489. 
461 Zhao, N., Zidan, A., Tawakkul, M., Sayeed, V.A., Khan, M., 2010. Tablet splitting:

462 Product quality assessment of metoprolol succinate extended release tablets. Int. J. Pharm. 401 (1-2), 25-31. 